\title{
Dossier Adaptation aux changements climatiques * Cette ambiguë adaptation au changement climatique
}

\author{
Olivier Godard \\ Économiste, CNRS, UMR 7176, École polytechnique, Route de Saclay, 91128 Palaiseau cedex, France
}

\author{
Mots-clés : \\ changement \\ climatique ; \\ adaptation ; \\ atténuation ; \\ négociations \\ internationales ; \\ cadrage économique
}

\section{Keywords:}

climate change;

adaptation;

mitigation;

international

negotiations; economic framing

\begin{abstract}
Résumé - L'article étudie la signification de la montée en visibilité du thème de l'adaptation. Après un examen des enjeux théoriques du concept à partir de la théorie des systèmes, son statut est analysé à la fois dans le discours des experts économistes et dans les négociations internationales engagées en vue d'un nouvel accord sur la politique climatique. Au-delà de la prise en charge de la part inéluctable de transformation du climat pour les trente prochaines années, le thème de l'adaptation a, dans le passé, servi à minorer l'ampleur des efforts de réduction des émissions à consentir et, à présent, il est le vecteur $\mathrm{d}^{\prime}$ un renoncement implicite à l'objectif de contenir l'augmentation de température à $2{ }^{\circ} \mathrm{C}$ au-dessus du niveau préindustriel. Cette signification prend appui sur le mode de représentation économique qui pose l'adaptation et l'atténuation comme deux moyens alternatifs équivalents pour une même fin, la maximisation d'une fonction d'utilité sociale intertemporelle. Diverses objections sont énoncées à l'encontre des hypothèses couramment faites dans ce contexte.
\end{abstract}

\begin{abstract}
Adaptation to climate change: so ambiguous. The paper considers the meaning of the increasing importance given to adaptation to climate change. First it examines the theoretical challenge of the concept from the systems theory standpoint. Paradoxically this leads to focussing attention on the values and structural features specific of the system considered rather than on the features of its environment. This outcome justifies the introduction of a key distinction between those features and components touching the identity of the system and those that are purely instrumental. The status of adaptation is then scrutinized both in the discourse of economic experts and in international negotiations that aim to set up a new climate agreement. Beyond the need to take account of the inescapable part of climate change over the next thirty years, the paper shows how in the past the notion of adaptation has been mobilized to downsize the pressing need to take immediate vigorous mitigation policies. At present, its seems that the rise of adaptation is the sign and vector of an implicit abandonment of the goal of containing climate change within the $2{ }^{\circ} \mathrm{C}$ increase range from pre-industrial levels. This meaning finds an appropriate vehicle in the economic mode of representation that considers mitigation and adaptation as two alternative means towards the same end, i.e. maximization of an intertemporal social utility function. Several objections are raised against the current assumptions made in that context.
\end{abstract}

\section{Introduction}

On nous laissait entrevoir un changement climatique pour dans longtemps ${ }^{1}$. De plus, rien n'était sûr, puisque toute l'affaire pouvait encore accoucher d'une augmentation modérée de la température moyenne globale de $1,5^{\circ} \mathrm{C}$ seulement. Certains voulaient alors surtout y voir

Auteur correspondant : olivier.godard@polytechnique.edu

* Cf. dans ce numéro, la présentation par la Rédaction du dossier «Adaptation aux changements climatiques ».

1 Cet article développe les idées avancées par l'auteur lors de la conférence invitée donnée le 8 octobre 2008 aux Journées annuelles de l'association Natures Sciences Sociétés - Dialogues, consacrées au thème : «Changement climatique : l'adaptation à l'ordre du jour». des avantages, comme le gain de terres cultivables, une plus grande productivité agricole et l'ouverture de nouvelles voies maritimes dans l'Arctique. Nous envisagions d'aborder confortablement le problème en raisonnant sur des actions doucement étalées sur plusieurs décennies. Et voilà que la réalité d'un changement climatique s'est imposée à nous : ce n'est plus seulement une spéculation; il est palpable. Certaines de ses manifestations sont à première vue pittoresques, comme l'avancée $\mathrm{d}^{\prime}$ un mois de la date des vendanges en France (Chuine et al., 2004; Seguin, 2007) ou la dérive d'icebergs géants. D'autres sont déjà plus inquiétantes, comme l'exposition des petites îles à la remontée du niveau de la mer ou la déstabilisation des sols en dégel dans le grand Nord, sans 
parler de l'accroissement de la gravité des événements extrêmes (tempêtes, sécheresses, canicules, inondations), bien repérés par les compagnies de réassurance. Toutefois, le constat du changement en cours ne suffit pas à en attribuer la cause aux activités humaines ; bien que l'accroissement de leurs effets soit notable, on ne saurait formellement attribuer cette évolution des dommages des catastrophes naturelles à l'accumulation atmosphérique des gaz à effet de serre (GES) émis par l'homme. Certes, mais les évolutions observées sont conformes aux effets attendus d'un changement climatique d'origine anthropique. L'imputation très probable d'une part significative de ce changement à l'homme est d'ailleurs affirmée par le rapport du GIEC de 2007. C'est en croisant les données d'observation et une modélisation numérique assise sur les principes fondamentaux de la physique que les chercheurs en sont venus à la conclusion qu'ils ne parvenaient pas à reconstituer l'évolution observée sans prendre en compte le rôle des GES émis par l'homme.

Tout se passe plus vite qu'il n'était prévu il y a encore quelques années, tant pour les manifestations physiques du changement de climat que pour le rythme d'accumulation des GES dans l'atmosphère. Le souffle de l'irréversibilité des transformations en cours contribue à ébranler les croyances ne voyant que progrès lumineux dans la trajectoire de l'humanité. L'inquiétude gagne, en premier lieu chez les scientifiques les plus au fait. Certains ne nous annoncent-ils pas un bouleversement de ce visage de la planète qui a permis le développement de la civilisation (Hansen et al., 2008), et cela pour une centaine de milliers d'années (Archer, 2008) ? En contrepoint, et peut-être pour les mêmes raisons, les démarches de dénigrement public des sciences du climat ont été portées en France et dans d'autres pays industriels, depuis la fin de l'année 2009, par une forte vague médiatique aussi peu nuancée qu'elle ne l'avait été auparavant lorsque les médias rivalisaient dans le déploiement d'images de catastrophes annoncées. Une idéologie climato-sceptique, qui ne date pas d'hier ${ }^{2}$, a manifestement trouvé dans cette période d'espoirs déçus par l'insuccès de la conférence de Copenhague une écoute et un marché satisfaisant les projets idéologiques de tout un chacun (Godard, 2010).

De fait, un certain niveau de changement climatique, peut-être modéré, peut-être déjà inéluctablement catastrophique, ne peut plus être évité, alors que rien n'était encore définitivement joué il y a 20 ans, lorsque le GIEC $^{3}$

\footnotetext{
2 Se reporter au livre d'Yves Lenoir (1992) et à l'analyse critique que j'en avais faite dans NSS (Godard, 1993), puis à celui de Bjorn Lomborg (2001).

${ }^{3}$ Groupe d'experts intergouvernemental sur l'évolution du climat, créé fin 1988 par l'Organisation météorologique mondiale et par le Programme des Nations unies pour l'environnement. Sur l'organisation de cette expertise encore unique, longtemps présentée comme un modèle d'organisation pour d'autres questions d'intérêt majeur pour le devenir commun
}

fut créé. Le climat des trente prochaines années est quasiment fixé, quelle que soit la vigueur des actions qui pourraient être entreprises pour atténuer le phénomène $d$ 'accumulation des GES (Parry et al., 2009). C'est à un terme plus lointain, à partir de 2050 et au-delà, que l'impact des politiques se fera sentir. En admettant que puisse être suivi un taux annuel de $3 \%$ de réduction des émissions de GES une fois passé un pic des émissions mondiales, ce pic ne devrait pas intervenir plus tard qu'en 2015 pour garder $50 \%$ de chances de maintenir l'augmentation de la température moyenne de la planète en dessous de $2{ }^{\circ} \mathrm{C}$ par rapport au niveau préindustriel ${ }^{4}$. Aussi n'est-il pas contestable qu'il faille se préoccuper dès aujourd'hui des mesures visant à faciliter l'adaptation des personnes, de l'appareil de production et des sociétés à cette part inéluctable, mais incertaine dans son expression localisée et son ampleur, de changement climatique. Cependant, il y a lieu de réfléchir sur cette évidence. D'abord car la précipitation est ici comme ailleurs mauvaise conseillère ; le contenu à donner à des politiques d'adaptation est en effet des plus délicat, notamment du fait de l'ampleur de l'incertitude qui prévaut quant à la traduction localisée des manifestations concrètes du changement de climat. Surtout, la thématique de l'adaptation a pris, dans le débat entre experts et dans les négociations internationales, une signification particulière qui déborde largement le souci opérationnel et sur laquelle il convient de s'attarder.

Cet article explore deux directions, qui ne sont d'ailleurs pas sans rapport. La première propose une réflexion sur le concept d'adaptation, en prenant appui sur le cadrage apporté en théorie des systèmes par le complexe d'interactions système/environnement (Godard, 1995 et 1997). La seconde élucide la signification ambiguë de la mise en avant du thème de l'adaptation au changement climatique dans la période charnière actuelle et souligne, contre le modèle économique standard, l'absence de symétrie entre prévention et adaptation au sein d'une politique climatique.

\section{Le paradoxe de l'adaptation}

La théorie de l'évolution a donné lieu à de vives controverses. L'une d'elles porte sur la manière de concevoir l'adaptation des organismes vivants à leur environnement. C'est Richard Lewontin qui a mis en évidence

de l'humanité, mais soudain violemment et injustement attaquée peu avant et peu après la conférence de Copenhague de décembre 2009, voir Godard (2001), Dahan Dalmedico (2007) et Encinas de Munagorri (2009).

${ }^{4}$ Deux degrés, c'est l'objectif qui avait été retenu par l'Union européenne et qui est mentionné par l'accord de Copenhague de décembre 2009, lequel accord n'organise aucunement l'action internationale de façon à avoir une chance raisonnable d'atteindre cet objectif. Voir Rogelj et al. (2010). 
de la façon la plus forte et la plus polémique le paradoxe surgissant du darwinisme classique :

«Le concept classique d'adaptation est l'idée qu'il y a un problème qui préexiste et qu'un organisme le résout en s'adaptant au problème. Par exemple, les nageoires sont une adaptation pour la nage, ce qui signifie que la nage était un problème avant que les poissons aient des nageoires. À regarder l'évolution de cette manière, on doit en déduire que la nage était aussi un problème pour les arbres!»

«En fait, les êtres ne commencent à rencontrer ces problèmes et à s'adapter que quand ils commencent à interagir avec le monde. Si bien que l'adaptation ne renvoie pas à un processus dans lequel les organismes vont au-devant de problèmes préexistants, parce que les organismes font surgir ces problèmes par leur existence et leur changement. Le vrai problème de l'évolution est que les organismes sont en construction constante de leurs propres mondes et créent les problèmes qu'ils ont ensuite à résoudre. »

«La sélection naturelle ne permet pas à des adaptations de résoudre des problèmes posés par la nature. La sélection naturelle manifeste l'amélioration de la manière dont les organismes interagissent avec le monde lorsqu'ils ont déjà commencé à interagir avec lui ${ }^{5}$. »

Pour la théorie des systèmes, le concept d'environnement est un concept relationnel. C'est aussi le terme second d'une relation : l'environnement est toujours l'environnement d'un sujet de référence (organisme, système, société) s'affirmant de lui-même ou identifié par un observateur. L'environnement n'est pas la nature, l'en-soi. Il comprend des éléments issus du monde biophysique qui font sens, à plusieurs titres, pour le sujet de référence. En tant que complément du sujet, l'environnement ne peut être que défini, construit par ce dernier. L'environnement d'une puce n'est pas celui d'un éléphant. En même temps, cet environnement constitue l'externalisé du sujet, à savoir ce dont ce sujet, de par son organisation, n'assume pas la reproduction comme loi nécessaire et quotidienne de son existence (Godard, 1984). D'où une béance dans la régulation qui donne corps à la problématique de l'adaptation au-delà du paradoxe initial exprimé par la question : comment peut-on penser le problème de l'adaptation d'un organisme ou d'un système à son environnement, puisque cet environnement ne lui préexiste pas et que c'est lui qui le fait surgir?

C'est là qu'il faut comprendre qu'une seconde relation s'ajoute à celle qui vient d'être décrite : si la première fait de l'environnement le terme conceptuellement second de la relation à un sujet, une hiérarchie inverse surgit du fait que l'environnement rassemble les conditions

${ }^{5}$ Lewontin, cité par Ridley (1996), (traduction O. Godard); voir la vidéo de R. Lewontin à l'URL : http:// blackwellpublishing.com:443/ridley/video_gallery/RL_What_ is_an_adaptation.asp. Voir aussi Lewontin (1978 et 2000). d'existence de ce sujet, organisme ou système. Et, logiquement, la condition d'existence précède l'existence. Bien qu'auto-organisé, un système ou un organisme n'est pas son propre créateur à partir du néant. Il faut alors enrichir la compréhension en posant trois notions distinctes :

- il y a d'abord le monde objectif, ses éléments et le tissu des interrelations entre ces derniers qui le fait tenir. On l'appelle la nature, le milieu ou la biosphère selon les circonstances et la visée. C'est l'être-là, disponible, le réservoir de ressources potentielles mais aussi de conditions, de contraintes et de perturbations potentielles;

- il y a ensuite l'environnement comme complément d'un sujet de référence, qui emprunte à la nature certains de ses éléments, de ses traits et de ses potentiels pour les intégrer au système de signification de ce sujet, sans les incorporer dans son fonctionnement interne, sa régulation ou son identité ;

- il y a enfin l'écocomplexe, métasystème englobant dont le sujet est, par son existence et son action, une composante active, alors même que ce sujet ne perçoit qu'un environnement qu'il prend pour une nature, c'est-à-dire un objet extérieur séparé et indépendant de lui.

Entrer dans la problématique de l'adaptation, c'est accepter de considérer une relation d'enchevêtrement (Godard, 1995 et 1997) dans laquelle un sujet doit, à un moment donné, se confronter à une réalité qu'il tient, à ce momentlà et du point de vue de l'action, pour extérieure à luimême, alors qu'il a coproduit cette réalité et, surtout, qu'elle est intégrée à son propre espace de signification comme composante de son environnement. Le temps de l'adaptation à l'environnement est celui où les œuvres du sujet lui reviennent comme une force extérieure qu'elles ne sont pas. Cette extériorité est localement et pragmatiquement vraie, mais ontologiquement fausse à deux titres : du point de vue de l'origine, puisque cet apparent donné extérieur résulte de l'action du sujet ${ }^{6}$; du point de vue de sa signification, c'est-à-dire de ses effets, parce que, s'il touche le sujet et fait sens pour lui, c'est en fonction de ce que ce sujet est. L'enjeu d'une adaptation réussie est de mettre en œuvre cette transformation des composantes et fonctions physiques du sujet qui tout à la fois permette le maintien du réseau bouclé de relations formant la base organisationnelle de l'identité du sujet comme sujet autonome et soit compatible avec le profil de contraintes, ressources et perturbations issues de l'environnement.

\footnotetext{
${ }^{6}$ Il existe certes des sources proprement externes de fluctuations et perturbations émanant de la nature et affectant l'environnement du sujet considéré : variations de l'activité solaire, météorites, mutations génétiques au sein du vivant, etc. Ce ne sont pas elles qui font la problématique environnementale.
} 
En appliquant ce schéma conceptuel, d'abord pertinent pour l'organisme dans son écologie mais de portée théorique générale, aux relations entretenues par l'humanité avec la planète Terre, il résulte que les sociétés contemporaines doivent d'abord discerner ce qui les constituent de façon essentielle, et qui doit être maintenu comme loi première, et ce à quoi elles veulent répondre sous la forme d'une adaptation de leurs composantes matérielles. La première démarche à engager est paradoxalement une démarche de réflexivité plus que de décryptage anxieux, hâtif et hasardeux des signes physiques du changement climatique. Selon que les sociétés se transformeront dans un sens ou dans un autre, selon les priorités qu'elles se donneront, selon ce qu'elles considéreront comme tolérable ou inacceptable, le contenu pratique et social de l'adaptation à opérer sera différent. Les problèmes que la transformation du climat pose reflètent donc tout autant la réalité des sociétés que celle du climat. Les énoncer demeure de la responsabilité de nos contemporains. "L'ultime règle des relations entre l'organisme et son environnement est que la nature physique de l'environnement, en tant qu'elle est pertinente pour les organismes, est déterminée par les organismes eux-mêmes » (Lewontin, cité par McMurry, 2003).

D'un point de vue dynamique, l'adaptation s'inscrit dans une histoire ouverte de coévolution. En dépassant la métaphore de l'organisme, la question soulevée pour l'humanité est celle de déterminer le cheminement entre les parcours qui se profilent, sans avoir la prétention de maîtriser le terme de l'aventure. C'est là que l'usage idéologique du thème de l'adaptation importe, car il a influé et influera sur la volonté collective d'agir pour prévenir le risque d'une évolution climatique catastrophique.

\section{L'idéologie de l'adaptation et la grande défausse}

\section{Le basculement rapide du "c'est trop tôt " au «c'est trop tard » versus le déni du risque}

D'une façon déjà entrevue dans d'autres circonstances historiques, nous sommes dans une période charnière de changement brutal des imaginaires de l'avenir : l'apocalypse est venue se glisser au côté du progrès rayonnant pour structurer les représentations. Ce phénomène n'affecte plus seulement des minorités plus ou moins illuminées, mais touche le cœur de l'élite scientifique et dirigeante (Barbault, 2006 ; Lebeau, 2005 et 2008; Férone, 2008; Weitzman, 2009). L'insouciance pour l'avenir, la conquête ou la défense brutale de rentes économiques et, de façon certes infiniment plus légitime, l'acharnement mis par des populations engoncées dans la misère à améliorer leurs conditions de vie, se sont révélés être les premières sources objectives de la perpétuation d'une évolution dangereuse des concentrations atmosphériques de GES depuis 1990, date de parution du premier rapport du GIEC. Ces forces actives d'inertie ont ruiné la possibilité que l'humanité détenait encore il y a 20 ans de s'épargner une entrée dans la zone de danger climatique majeur.

Aujourd'hui, le changement brutal d'imaginaire de l'avenir est en passe d'avoir le même effet anesthésiant et mortifère sur la volonté d'influer sur le cours des choses. La découverte d'un avenir climatique préoccupant et potentiellement réellement catastrophique a suscité deux représentations au contenu sémantique opposé, mais aux effets convergents sur la propension à agir : pour la première, nous serions passés subrepticement, mais presque d'un coup, du «c'est trop tôt pour se préoccuper sérieusement de ce danger à long terme tellement incertain » au «c'est déjà trop tard pour empêcher l'essentiel de ce qui doit se produire $»^{7}$; pour la seconde, l'insuccès de la conférence de Copenhague confirme que la cause était frelatée, qu'on avait affaire à une fausse science, un mythe (Galam, 2008; Rittaud, 2010), un dogme (Allègre, 2010; Ewald, 2010), une idéologie "réchauffiste», que sais-je encore. La conviction d'être confronté à une inéluctable altération climatique et la logique du déni du risque convergent ici pour rendre insignifiante, voire inconvenante et immorale, toute idée de politique de prévention du risque climatique.

Les partisans de l'inaction climatique ont utilisé toutes sortes de manœuvres depuis 20 ans, depuis la dénégation des bases scientifiques de la « théorie » du changement climatique (Godard, 1993, 2007a et 2010) jusqu'à la présentation de la géo-ingénierie comme une panacée pouvant sauver le mode de vie occidental (américain) et la «civilisation » par une technologie enrayant la dérive de la machine climatique, en passant par la dénonciation du fonctionnement stalinien du GIEC (Allègre, 2006), la minoration des dommages climatiques ou la présentation catastrophiste des coûts économiques des politiques «d'atténuation ». Aujourd'hui, c'est le couple paradoxal associant déni et fatalité du changement climatique qui sert leur cause. Face à l'inéluctable ou face à un mythe, en effet, à quoi bon gaspiller les moyens disponibles dans un combat sans issue?

Ainsi ne resterait-il plus à l'humanité qu'à s'adapter à la nouvelle donne climatique sans prétendre en changer le cours. C'est la position défendue par

\footnotetext{
7 Selon un sondage du Guardian auprès de 260 scientifiques (Adam, 2009), plus de la moitié affirment qu'il serait possible de respecter l'objectif des $+2{ }^{\circ} \mathrm{C}$, mais la plupart pensent que $1^{\prime}$ humanité ne saura pas éviter une augmentation de 3 à $4{ }^{\circ} \mathrm{C}$ d'ici la fin du siècle. De plus, la recherche paléo-climatique suggère que la sensibilité climatique à long terme pourrait être le double de la sensibilité à court terme considérée jusqu'à présent (Hansen et al., 2008).
} 
Henri Atlan (2010) : «Plutôt que de tenter de prévenir des risques globaux incertains par des mesures globales à l'efficacité tout aussi incertaine, mieux vaut résoudre les problèmes localement en corrigeant ce qui peut l'être et en s'adaptant à ce qui ne peut pas être évité à court terme par des mesures d'urbanisation et de déplacements de populations si nécessaire. »

\section{La montée en visibilité de la thématique « adaptation », voile du renoncement à l'objectif des $2{ }^{\circ} \mathrm{C}$}

Les enjeux de l'adaptation ont été, nous disait Michel Damian (2007), injustement occultés par l'expertise, par le débat public et par les négociations internationales. Si tant est que cela soit $\mathrm{vrai}^{8}$, la montée en puissance de cette thématique est aujourd'hui non seulement le témoin, mais bien l'accompagnateur, voire le vecteur involontaire? - de ce basculement de l'imaginaire vers la résignation. Elle fait certes écho à la découverte de la réalité déjà perceptible du changement de climat et à la prise de conscience des inerties en jeu, qui font que les phénomènes climatiques des trente prochaines années, quelle qu'en soit l'origine, seront très peu sensibles aux actions qui pourraient être entreprises pour réduire le rythme d'accumulation des GES dans l'atmosphère. Toutefois, de façon voilée, elle habille et disculpe le manque de volonté politique internationale dans la maîtrise des émissions de GES ${ }^{9}$.

Naturellement, le message du renoncement n'est le message explicite ni des scientifiques du GIEC, qui ne font aucune recommandation, ni de tous ceux qui demandent qu'on accorde une plus grande attention aux politiques d'adaptation. Le discours politique dominant est qu'il incombe aux gouvernements et aux différents acteurs (entreprises, collectivités territoriales) d'entreprendre tout à la fois réduction des émissions et politiques d'adaptation (Stern, 2007; Lecocq et Shalizi, 2007). Damian (2007) voit même dans la revalorisation

\footnotetext{
${ }^{8}$ La thèse de l'occultation est excessive à la lecture des textes internationaux. La convention «climat» de 1992 mentionnait l'adaptation à plusieurs reprises. En 1994, le GIEC avait publié des lignes directrices sur l'évaluation de la vulnérabilité et des besoins d'adaptation. En 1995, la conférence de Berlin énonçait des recommandations sur les mesures d'adaptation. Le second rapport du GIEC, en 1996, soulignait l'importance de politiques « sans regrets» d'adaptation, etc. (Jamieson, 2005).

9 Un récent rapport de l'IDDRI pose ainsi l'équation : «Si la majeure partie de l'attention se focalise encore sur le volet mitigation, l'adaptation prend de plus en plus d'ampleur au fur et à mesure que l'on se rend compte des difficultés à définir et à atteindre des objectifs de mitigation à la fois partagés, réalistes et suffisamment ambitieux. La prise de conscience d'une certaine irréversibilité des changements engagés joue ainsi en faveur d'une attention croissante aux besoins et enjeux de l'adaptation » (Magnan et al., 2009, p. 2).
}

de l'adaptation un moyen de crédibiliser les politiques de réduction des émissions, mais sans expliquer d'où viendrait cet effet en retour vertueux.

Cette pétition de principe ne convainc guère, au regard des signes multiples témoignant du refus de la communauté internationale de s'engager avec détermination dans la réduction des émissions de GES. La conférence de Copenhague a cruellement déçu tous ceux qui attendaient que soit mis en place un régime de type Kyoto étendu aux principaux pays émetteurs, industriels, émergents ou en développement, et profilé en fonction d'objectifs à différents horizons (2020, 2050). Elle a en revanche accepté le principe d'aides financières de court et moyen terme au profit des pays en développement, en particulier pour lutter contre la déforestation. Dans les négociations préparatoires de cette conférence, la priorité des grands pays émergents comme la Chine et l'Inde, rassemblant autour d'eux le Groupe des 77, avait manifestement été d'obtenir de tels transferts davantage que de s'engager eux-mêmes sur un profil de maîtrise de leurs propres émissions, même s'ils ont finalement concédé une déclaration non contraignante d'objectifs définis en termes relatifs (intensité carbone du PIB) proches des tendances «au fil de l'eau ». Leur refus s'alimentait à l'invocation ferme de la responsabilité historique des pays développés et de leur propre droit au développement, qui, à leurs yeux, primerait sur toute autre considération. Or, la maîtrise des émissions de ces pays, qui pèsent pour plus de la moitié des émissions mondiales courantes, celles sur lesquelles on peut agir, est une composante indispensable de l'effort international requis pour tenir l'objectif des $2{ }^{\circ} \mathrm{C}$.

Du principe à la concrétisation, il y a plus de chemin à parcourir que de la coupe aux lèvres. Les transferts financiers qui étaient revendiqués devaient favoriser tout à la fois le développement économique des pays du Sud, leur adaptation aux impacts du changement climatique et l'obtention de réductions d'émissions par rapport à un scénario tendanciel. Les montants sollicités étaient considérables : ces pays demandaient que leur soit transféré annuellement, au titre de la politique climatique, entre 0,5 et $1 \%$ du PIB des pays développés, soit entre 200 et 400 milliards de dollars par an, de façon additionnelle à l'aide au développement classique (UNFCC, 2008; Drouet, 2009). De plus, ils demandaient aux pays développés, pris comme un tout, de réduire leurs propres émissions de GES dans une fourchette de 25 à $40 \%$ d'ici 2020 et de 90 \% d'ici 2050, par rapport à 1990 .

Face à ces demandes, 1'Union européenne proposait de maintenir un prélèvement de $2 \%$ sur les crédits du mécanisme de développement propre (MDP) pour alimenter un fonds d'adaptation des pays en développement, pour un montant annuel compris entre 0,2 et 0,7 milliards de dollars (UNFCC, 2008). Les États-Unis consentaient à envisager une quasi-stabilisation de leurs émissions au 
niveau de 1990 d'ici 2020 et des réductions fortes à long terme (2050), tandis que d'autres pays comme le Canada ou le Japon rechignaient, sans parler de la résistance opposée au sein de l'Union européenne par les nouveaux États membres de l'Est et en premier lieu la Pologne, mais aussi par l'Italie. Face à cela, l'accord de Copenhague se présente comme un demi-succès d'estime, puisqu'il mentionne une enveloppe de transferts de 30 milliards de dollars d'ici 2012, devant augmenter jusqu'à 100 milliards en 2020. Les sources et la répartition de ce financement ne sont toutefois pas davantage précisées.

Nous sommes manifestement éloignés de l'état de mobilisation de tous les pays du monde qui serait requis pour contenir les concentrations à un niveau inférieur à $450 \mathrm{ppm}$ de $\mathrm{CO}_{2} \mathrm{eq}^{10}$. Implicitement, la communauté internationale a déjà consenti à se livrer à l'aventure climatique, en contradiction avec l'objectif qu'elle s'est fixé en 1992 dans la Convention-cadre sur les changements climatiques : celui d'éviter une interférence dangereuse avec le climat de la planète, en contradiction, également, avec l'objectif cible des $2{ }^{\circ} \mathrm{C}$ mentionné dans l'accord de Copenhague. La place réservée désormais à l'adaptation est l'expression subliminale de ce renoncement. Quant aux attentes financières du Sud, déjà sévèrement recadrées à la baisse après Copenhague, elles pourraient déboucher sur la même déconvenue que le projet de réduction drastique des émissions : il n'y a guère de raison que l'absence de volonté forte ne soit pas un facteur commun.

\section{Ce qui distingue l'adaptation de l'atténuation}

Deux caractéristiques différencient les approches d'adaptation des stratégies de réduction des émissions nettes («l'atténuation »). La réduction des émissions vise à influer sur la qualité d'un bien collectif planétaire; il s'agit d'une production décentralisée d'un bien collectif, qui est donc très exposée au risque du passager clandestin et en conséquence très demandeuse d'une coordination internationale ferme, mais difficile à obtenir. Par ailleurs, l'inertie des phénomènes sous-tendant le changement climatique impose, pour éviter la zone de danger majeur, l'engagement immédiat d'actions fortes à impulser jusqu'en 2050. Cet engagement doit a priori mobiliser l'ensemble des émetteurs de GES.

En revanche, l'adaptation relève principalement de la protection ou de la production de biens privés qui devraient être spontanément prises en charge par les acteurs

\footnotetext{
${ }^{10} \mathrm{CO}_{2}$ eq signifie équivalent $\mathrm{CO}_{2}$; il s'agit d'une mesure commune à l'ensemble des gaz à effet de serre, établie à partir de leur pouvoir de réchauffement. La référence la plus fréquemment retenue, par convention, est un horizon de 100 ans pour définir les équivalences entre gaz.
}

économiques et politiques concernés. L'essentiel des bénéfices des actions d'adaptation sont privés, c'est-à dire qu'ils bénéficient à ceux qui les engagent et à eux seulement : quitter une zone littorale menacée par la mer, changer de culture agricole pour s'adapter à une nouvelle donne pluviométrique, par exemple, relèvent de décisions individuelles ou locales qui concernent directement les intéressés. La dimension collective de l'adaptation n'apparaît que de façon périphérique à la faveur de trois aspects : l'équité de la distribution des efforts à accomplir (besoins d'aides de la part de populations n'ayant pas les ressources pour s'adapter); le manque d'information et de compétences; l'incidence négative de certaines actions d'adaptation sur le problème climatique lui-même via les émissions de GES qu'elles engendrent.

S'agissant du calendrier de l'action, on peut certes distinguer deux types d'adaptation : une adaptation réactive qui se met en place après des événements climatiques particuliers ou en s'ajustant graduellement à des évolutions physiques; une adaptation anticipative, comme celle qui mise sur la recherche et le développement pour mettre au point de nouvelles semences ou qui programme les travaux publics nécessaires pour relever le niveau des digues ou renforcer l'enrochement du littoral, ou encore pour déplacer progressivement certaines populations vulnérables. Même dans ce second cas, hormis pour ce qui concerne la recherche, la gestion forestière ou la protection des côtes, qui demandent d'engager l'action très en avance par rapport à la réalité des phénomènes, il n'y a guère lieu de lancer des actions de grande envergure qui projetteraient d'anticiper de plusieurs décennies des évolutions dont la concrétisation demeure très incertaine à l'échelle locale.

Au total, le centre de gravité des stratégies d'atténuation concerne une action immédiate et coûteuse à engager par les pollueurs pour un bénéfice collectif planétaire - la réduction de tous les risques dérivés du changement climatique -, tandis que le centre de gravité de l'adaptation est une action largement différée dans un temps assez éloigné, relevant de l'initiative privée des «victimes » pour des actions procurant des bénéfices privés, mais n'apportant à chaque fois que des réponses partielles et locales à tel ou tel aspect du changement climatique.

\section{La rhétorique minorante de l'adaptation}

Depuis que la question du changement climatique est devenue une question politique, la mise en avant de l'adaptation a toujours été mobilisée dans les jeux d'influence tant entre experts que dans les relations politiques internationales. 


\section{Du côté des experts}

En mettant en avant l'adaptation, certains experts proches des pays les plus émetteurs proposaient implicitement de renvoyer l'action aux calendes grecques, faisaient miroiter l'absence de besoin d'une coordination internationale forte, valorisaient les solutions de marché, en particulier pour le développement des couvertures assurantielles et des partenariats public-privé, et surtout proposaient de céder le mistigri aux « victimes » puisque, selon leurs vues, il appartiendrait à chacune d'elles de choisir comment se prémunir vis-à-vis des dommages climatiques qui pourraient l'affecter.

Les partisans d'une action climatique modeste, écartant tout effort significatif d'atténuation (Nordhaus, 1995), ont d'abord mobilisé l'adaptation comme moyen de minorer l'évaluation ex ante des dommages climatiques à venir, et donc la portée des efforts de prévention à consentir dans l'immédiat et dans le futur proche. Cet usage s'est appuyé sur des arguments empruntés à des registres différents, allant de l'argumentation scientifique sérieuse à la position idéologique confinant au grotesque. À l'intérieur de ce spectre, on trouvera les analyses d'économistes ayant pignon sur rue (Rosenzweig et Parry, 1994; Fankhauser, 1995; Tol et al., 1998; Tol, 2005), les positions d'idéologues (Lomborg, 2001) et celles de responsables politiques ${ }^{11}$. Par exemple, estimant que l'adaptation est de loin la voie la moins coûteuse, Richard Tol adressait récemment des critiques sévères non seulement au rapport Stern (Tol et Yohe, 2006), mais aussi aux objectifs de réduction des émissions adoptés par l'Union européenne en référence à $1^{\prime}$ objectif des $2{ }^{\circ} \mathrm{C}$, jugé totalement arbitraire et sans fondement économique (Tol, 2007).

L'opération intellectuelle servant de base à l'usage minorant de l'adaptation a deux composantes. La première consiste à voir dans l'adaptation un facteur de diminution ou de neutralisation des dommages «bruts ». Par exemple, si les digues sont remontées de $40 \mathrm{~cm}$, l'impact d'une remontée des mers de $30 \mathrm{~cm}$ sera neutralisé, alors que le dommage créé sera important dans le cas contraire. Et si l'on déplace à temps les populations qui habitent dans les zones littorales les plus exposées, les dommages économiques potentiels seront à nouveau considérablement réduits. Il en va de même si le génie génétique met au point des semences beaucoup plus résistantes au stress hydrique et si la recherche pharmaceutique invente de nouveaux médicaments pour soigner les populations

\footnotetext{
11 Le sénateur américain du Texas, Joe Barton, s'est ainsi exprimé le 25 mars 2009 devant la Chambre des représentants des États-Unis : «Adapting is a common, natural way for people to adapt to their environment. I believe that the earth's climate is changing but I think it's changing for natural variation reasons. I think mankind has been adapting to climate as long as man has walked the earth" (Barton, 2009).
}

qui seront nouvellement affectées par des maladies diffusées par la migration de vecteurs pathogènes dont l'écologie aura été modifiée. Etc. Dans ce dossier, le sujet le plus important est l'impact sur la production alimentaire et donc sur la productivité agricole. Comme l'analysait Nordhaus (1995), les «adaptationnistes » considéraient que le climat pouvait engendrer des chocs à court terme, mais que l'effet de ces derniers serait annulé à plus long terme par l'adaptation, si bien que sur plusieurs décennies on ne devait escompter aucun impact économique négatif, une fois absorbés les coûts de transition. Et cela, sans compter avec l'impact fertilisant de l'accroissement de la concentration atmosphérique en $\mathrm{CO}_{2}$.

La seconde composante consiste à présenter l'adaptation et l'atténuation comme deux stratégies permettant d'atteindre des résultats équivalents, comme deux moyens alternatifs pour une même fin. Cela n'est possible qu'en adoptant le cadrage utilitariste anthropocentrique qui sert de base théorique à l'analyse économique normative la plus courante, qu'on appelle néoclassique. Pour cette dernière, toutes les différences concrètes sont effacées par un jugement en « utilité », fonction abstraite construite à partir des relations de préférences attribuées aux individus, mais encadrées par des contraintes de « rationalité » (transitivité des préférences; utilité marginale décroissante; préférence pure pour le présent). Le problème posé devient alors celui de la meilleure combinaison entre prévention et adaptation (Jepma et Munasinghe, 1998; Tol, 2005), la meilleure étant celle qui minimise le coût intertemporel pour une collectivité dont l'intérêt général est représenté sous la forme d'une fonction d'utilité sociale agrégeant les préférences individuelles.

Dans la mesure où les coûts de l'atténuation sont à consentir dès aujourd'hui, et de façon croissante durant les 40 ans à venir, et où l'essentiel des coûts de l'adaptation sont reportés à des temps plus éloignés en fonction des manifestations du changement climatique, on comprend sans peine que le jugement sur le bon équilibre de la combinaison va dépendre de façon cruciale du taux d'actualisation utilisé, comme l'a montré la controverse suscitée par le rapport Stern (2007) [Godard, 2007b et 2008b], mais aussi des hypothèses concernant l'évaluation des dommages et l'évolution des prix relatifs entre actifs naturels et biens productibles (Sterner et Persson, 2008) : la totalité de l'écart entre les recommandations totalement inverses de Stern (2007) et de l'un de ses critiques les plus vifs, Nordhaus (2007b), s'explique à partir des choix respectifs de ces deux auteurs concernant le taux d'actualisation (1,4\% pour le premier ; $5,5 \%$ pour le second). Les économistes qui utilisent à la fois des taux courants d'actualisation ( 5 à $8 \%$ par an) sur des périodes de 200 ans et des fonctions de dommages fondées sur des valeurs modérées d'accroissement de température (entre 2 et $3{ }^{\circ} \mathrm{C}$ ), induisant un niveau agrégé modeste 
de dommages, concluent généralement que l'essentiel de l'effort à consentir pour le climat doit être reporté de plusieurs décennies, c'est-à-dire relever de stratégies d'adaptation. Au nom de l'efficacité et pour le bien collectif ${ }^{12}$.

De plus, pour les régions et populations les plus vulnérables, c'est-à-dire la moitié de l'humanité, la meilleure adaptation serait à trouver dans l'accélération de la croissance économique, puisque cela réduirait leur dépendance vis-à-vis des phénomènes naturels (Schelling, 1997; Lomborg, 2001), quitte à ce que cette croissance accélérée amplifie objectivement le danger climatique : l'agriculture, l'activité la plus liée aux événements climatiques, représente $2 \%$ du PIB des pays développés comme les États-Unis, mais $30 \%$ de celui des pays en développement.

\section{Du côté des négociations internationales}

Les négociateurs des pays du Sud ont bien perçu les implications du message des experts prônant l'adaptation, et ils ont vite retourné l'argument pour faire de leurs besoins d'adaptation la source d'une obligation de transferts financiers importants de la part des pays historiquement les plus pollueurs et les plus riches. Forçant la porte de la négociation internationale autour de ce thème, ils ont réussi à imposer qu'il devienne un élémentclé d'un accord global. Cela a changé l'équilibre de cette négociation, en éloignant les positions des parties des deux bords : alors que la priorité affichée par les pays du Nord est d'obtenir une large coopération internationale sur une trajectoire de réduction globale des émissions, et en particulier des engagements sérieux des pays du Sud sur ce plan, la priorité de ces derniers est de soutirer des flux financiers conséquents aux pays du Nord pour leur permettre de s'adapter, eux qui seront les principales victimes des dommages climatiques, et plus généralement de financer leur modernisation technologique.

\section{Pour une critique des fondements économiques et éthiques d'une stratégie misant sur l'adaptation}

La critique du rôle prééminent que certains veulent voir jouer à l'adaptation comme substitut des politiques

\footnotetext{
12 En 1995, Nordhaus déterminait un niveau de départ optimal pour une taxe sur le $\mathrm{CO}_{2}$ à $1,25 \$ / \mathrm{tCO}_{2}$, devant croître très progressivement jusqu'à 5 \& en 2100 (Nordhaus, 1995). Dix ans plus tard, après avoir revu tous ses calculs avec le modèle DICE, il aboutissait à une valeur de 6,4 \$/tCO 2 comme valeur optimale en 2007, évoluant vers 23 \$ en 2050 (Nordhaus, 2007a). En France, la commission Quinet, réunie à la demande du gouvernement pour déterminer la valeur tutélaire du $\mathrm{CO}_{2}$ à prendre en compte dans les choix publics, a préconisé une valeur de $32 €$ en 2010, croissant jusqu'à $100 €$ en 2030 (CAS, 2009).
}

d'atténuation qui leur incombent doit se déployer sur le terrain factuel et sur le terrain éthique.

D'abord, cette manière de présenter adaptation et atténuation comme des moyens alternatifs pour réaliser une même fin ne s'accorde pas avec une conception du développement durable qui ne se satisfait pas de l'hypothèse de substituabilité large entre capital productif, capital humain et capital naturel, mais pose une contrainte de préservation intertemporelle soit d'un capital naturel critique, soit du capital naturel en tant que totalité (Vivien, 2005). Elle ne s'accorde pas davantage avec une approche de viabilité ou encore avec les conceptions qui reconnaissent une valeur $\mathrm{d}$ 'existence intrinsèque aux êtres naturels, toutes conceptions qui obligeraient le raisonnement à ne pas se contenter de comptabiliser les effets dommageables du changement climatique pour le bien-être économique des humains ou pour la croissance économique.

Il faut aussi réfléchir à l'effet d'effacement ou d'atténuation du dommage climatique attribué à l'adaptation. Relever des digues ou changer de semences sont des actions qui peuvent être perçues comme suffisamment extérieures pour ne pas altérer l'essence du fonctionnement culturel ou social d'une société, ce qui touche à ses valeurs essentielles. Sauf pour les semences, si le changement technique est le vecteur d'un bouleversement des rapports économiques induisant, via le brevet, une dépendance annuelle nouvelle à l'approvisionnement auprès de firmes multinationales. Mais qu'en est-il du déplacement massif de populations vivant sur le littoral ou dans des zones vulnérables à l'invasion de l'eau de mer? C'est certainement mieux que de les observer passivement se noyer. Mais peut-on établir une équivalence en utilité avec une réduction du risque à sa source? Pour forcer le trait, citons cette forme d'adaptation anticipative qui fut pratiquée par la médecine américaine au profit (?) des populations environnantes des atolls ayant servi aux essais nucléaires atmosphériques : « Après les essais atomiques, les médecins américains ont pratiqué à la chaîne des ablations de thyroïdes. Ils préféraient s'en débarrasser avant qu'un cancer ne se déclare» ([n.s.], 2009). Ces populations n'auraient-elles pas préféré ne pas avoir été exposées à la radioactivité?

On est ainsi conduit à préconiser un double regard sur les mesures d'adaptation, en distinguant celles qui peuvent être mises en balance avec un effort de réduction des émissions dans un calcul d'ensemble et celles qui ne peuvent être éventuellement considérées que dans un état de nécessité où l'irrémédiable a déjà été commis. Et, dans les deux cas, en remettant le point de vue utilitariste à sa place, qui n'est pas d'être la matrice générale des choix collectifs, il convient de s'interroger sur les implications des actions envisagées : ne sont-elles que des instruments ne remettant en cause rien d'essentiel des valeurs collectives, du pacte social ou de l'équilibre 
des rapports économiques, ou au contraire sont-elles susceptibles d'y porter sévèrement atteinte?

$\mathrm{D}^{\prime}$ autres critiques visent le jeu d'hypothèses qui soustendent le raisonnement économique courant en la matière (Godard, 2007b) :

(1) la plupart des économistes font l'hypothèse d'une croissance économique se poursuivant à l'avenir de façon indéfinie, ce qui les conduit à tenir pour assuré que les générations futures seront globalement beaucoup plus riches que les générations actuelles, quelles que soient les vicissitudes climatiques ${ }^{13}$. Rien n'est moins sûr pour le long et le très long terme. Du fait de la formidable inertie du climat, rien ne garantit que les générations futures n'aient pas à affronter à la fois un effondrement économique et une évolution catastrophique du climat. Or, les générations actuelles ont plus d'influence sur l'évolution du climat que sur la situation économique à long terme ; le souci pour les générations futures devrait d'abord se manifester dans une politique d'évitement d'évolutions dangereuses du climat;

(2) l'hypothèse selon laquelle la capacité d'adaptation d'une société est directement proportionnelle à l'importance de son PIB implique mécaniquement, en intégrant l'hypothèse (1), que les générations futures auront d'autant plus de capacités à s'adapter qu'elles seront éloignées de nous. Or, cette capacité dépend d'abord, c'est une lapalissade, des capacités mobilisables qui auront été préalablement formées ou accumulées, par une politique antérieure avisée d'investissement dans de telles capacités - concept de résilience - et dans une diversité suffisante de moyens pour satisfaire des besoins donnés, comme les transports ou l'accès à l'eau - concept de flexibilité. Elle dépend également du surplus économique disponible, à travers l'investissement, pour réorganiser la base physique du fonctionnement économique bien davantage que du niveau absolu de richesse économique. De ce surplus, rien ne nous permet de dire que les générations futures seront plus dotées que les générations actuelles. L'exemple des États-Unis est là pour montrer que richesse et capacité d'adaptation ne vont pas forcément de pair : ce pays ne met-il pas en avant l'inertie de ses structures urbaines et sa dépendance majeure au transport automobile?

(3) l'hypothèse d'absence d'effet cumulatif ou systémique des événements climatiques sur le fonctionnement économique et social manifeste un postulat de marginalité de l'incidence climatique ${ }^{14}$. Or, les systèmes socio-

\footnotetext{
${ }^{13}$ Le rapport Stern (2007) ne fait pas exception en postulant un taux de croissance par tête de 1,3\% sur l'ensemble de l'horizon considéré, jusqu'en 2200, même en cas de changement climatique catastrophique.

14 Pour l'étude de l'hypothèse inverse, voir les travaux de S. Hallegatte sur les incidences macroéconomiques possibles d'événements climatiques extrêmes à répétition (Hallegatte, 2008 ; Hallegatte et Ghil, 2008).
}

écologiques peuvent absorber de petits chocs espacés, mais de petits chocs répétés sont susceptibles d'avoir des effets cumulatifs et une succession de chocs importants est susceptible de faire plonger ou de maintenir des sociétés dans le sous-développement. Il faut également compter avec les interdépendances systémiques entre plusieurs types de dommages : perturbation du cycle de l'eau, menaces sanitaires, moindre production agricole, événements extrêmes à répétition pourront se combiner pour devenir des obstacles sérieux au développement et multiplier les trappes à pauvreté. L'idée de limite à l'adaptation s'impose ici, qui souligne le lien étroit de complémentarité entre les options viables d'adaptation et les politiques d'atténuation : ce sont ces dernières qui, en permettant d'éviter un emballement climatique, ouvrent un espace viable pour des stratégies d'adaptation ;

(4) le fait que les générations futures puissent disposer de capacités d'adaptation ne justifie pas, sur le plan éthique, qu'on leur transfère le fardeau. La mise en forme du choix par le modèle économique standard présente les générations actuelles comme libres de leurs choix quant à l'étendue du « sacrifice ${ }^{15}$ » qu'elles sont prêtes à consentir pour le bien des générations suivantes, tandis que ces dernières sont, dans le raisonnement, tenues de s'adapter de façon optimale aux conditions climatiques que les premières leur imposeront. Une telle asymétrie de traitement est choquante. Ne faudrait-il pas intégrer le libre arbitre et le droit à l'erreur des générations futures, sans préjuger de leurs choix et même en considérant la possibilité qu'elles refusent l'adaptation sur laquelle leurs ancêtres voudraient parier (Godard, 2008a) ? La recherche de l'efficacité économique a ici bon dos, puisqu'elle couvre de sa respectabilité un transfert du fardeau aux générations qui auront à subir les dommages (Gardiner, 2006 ; Gosseries, 2006), alors que les générations actuelles sont les seules à bénéficier des contreparties positives de ces dommages sous la forme d'un niveau de vie assurant voyages, confort dans l'habitat et une alimentation fortement carnée.

Face à un changement climatique inéluctable, mieux vaut prendre des mesures pour faciliter l'adaptation de l'économie, de la société et des personnes que de demeurer vulnérable au nom de la conservation de façons de vivre et de faire familières. Cependant, pour la part de ce changement qui n'est pas inéluctable, les propositions visant à miser principalement sur des stratégies

\footnotetext{
15 Il faudrait s'interroger sur la propension des économistes du climat à employer si fréquemment le terme «sacrifice » pour désigner ce que demandent aux générations actuelles des politiques d'atténuation qui ne sont qu'un moyen partiel de leur imputer les coûts de leurs modes de vie qui, sinon, seront supportés, en nature, par d'autres qu'eux. Lorsqu'on somme un débiteur de payer sa dette, dit-on qu'on lui demande un sacrifice?
} 
d'adaptation doivent être accueillies avec la plus grande circonspection. Elles reposent généralement sur un ensemble d'hypothèses que les faits ne confirment pas ou sur des postulats normatifs et éthiques dont la pertinence et la valeur ne sont pas assurées face à un problème aussi majeur que le changement climatique. Au-delà des apparences, l'aspect le plus crucial de tout raisonnement sur l'adaptation n'est pas de discerner les caractéristiques du monde extérieur auxquelles les sociétés devraient s'adapter, mais d'éprouver par la réflexivité et le débat public ce que sont les éléments essentiels de l'organisation collective qui ne doivent pas être mis en jeu dans l'adaptation et ce qui peut l'être. Un tel exercice serait un test très significatif de la ligne réelle de partage entre solidarité et responsabilité individuelle dans nos sociétés.

Finalement, que ce soit sous la forme de l'atténuation ou de l'adaptation, la perspective du changement climatique appelle les générations actuelles à consacrer des ressources importantes à l'investissement, afin de permettre tout à la fois la constitution de capacités d'adaptation future à des changements climatiques dont la réalité locale est et demeurera marquée par $1^{\prime}$ incertitude ${ }^{16}$ et une transformation de la base technique et physique des sociétés pour réaliser cette économie à bas profil en carbone que la menace climatique et la raréfaction pétrolière commandent de promouvoir à assez brève échéance. Il reste à inventer les conditions politiques et économiques de cette affectation d'une part accrue du produit annuel à l'investissement, car sa contrepartie, à niveau de croissance donnée, est une réduction relative de la part allouée à la consommation courante. Continuerons-nous à refuser l'obstacle?

\section{Références}

[n.s.], 2009. Les cobayes du Dr Folamour, Le Monde, 23 juin.

Adam, D., 2009. World will not meet 2C warming target, climate change experts agree, The Guardian, 14 April.

Allègre, C., 2006. Le droit au doute scientifique, Le Monde, 27 octobre.

Allègre, C., 2010. L'Imposture climatique, ou la Fausse Écologie, Paris, Plon.

Archer, D., 2008. The Long Thaw: How Humans Are Changing the Next 100,000 Years of Earth's Climate, Princeton, Princeton University Press.

Atlan, H., 2010. La religion de la catastrophe, Le Monde, 27 mars.

Barbault, R., 2006. Un éléphant dans un jeu de quille, Paris, Le Seuil.

Barton, J., 2009. Testimony, House Energy and Commerce Committee Hearing, Washington, DC, 25 March (http:// republicans.energycommerce.house.gov/news/PRArticle. aspx?NewsID=6912, consulté le 24 avril 2009).

\footnotetext{
${ }^{16}$ Cela implique notamment d'accroître les capacités des réseaux, infrastructures et unités de production qui auront à faire face à une plus grande variété de situations.
}

Centre d'analyse stratégique (CAS), 2009. La Valeur tutélaire du carbone : rapport de la commission présidée par A. Quinet, Paris, La Documentation française.

Chuine, I., Yiou, P., Viovy, N., Seguin, B., Daux, V., Le Roy Ladurie, E., 2004. Grape harvest dates and temperature variations in France since 1370, Nature, 432, 289-290, doi :10.1038/nature432289a.

Dahan Dalmedico, A., 2007. Le régime climatique, entre science, expertise et politique, in Dahan Dalmedico, A. (Ed.), Les Modèles du futur. Changement climatique et scénarios économiques : enjeux scientifiques et politiques, Paris, La Découverte, 113-139.

Damian, M., 2007. Il faut réévaluer la place de l'adaptation dans la politique climatique, Natures Sciences Sociétés, 15, 4, 407410.

Drouet, A., 2009. Financer l'adaptation aux changements climatiques : ce que prévoit la Convention-cadre des Nations unies sur les changements climatiques, Paris, Mission climat de la Caisse des dépôts.

Encinas de Munagorri, R., 2009. Expertise et gouvernance du changement climatique, Paris, LGDJ.

Ewald, F., 2010. Apologie de Claude Allègre, Les Échos, 2 mars.

Fankhauser, S., 1995. Valuing Climate Change: The Economics of the Greenhouse Effect, London, Earthscan.

Férone, G., 2008. 2030 : le krach écologique, Paris, Grasset.

Galam, S., 2008. Les Scientifiques ont perdu le Nord : réflexions sur le réchauffement climatique, Paris, Plon.

Gardiner, S.M., 2006. Protecting future generations: Intergenerational buck-passing, theoretical ineptitude and a brief for a global core precautionary principle, in Tremmel, J.C. (Ed.), Handbook of Intergenerational Justice, Cheltenham (UK), Edward Elgar, 148-169.

Godard, O., 1984. Autonomie socio-économique et externalisation de l'environnement : la théorie néo-classique mise en perspective, Économie appliquée, XXXVII, 2, 315-345.

Godard, O., 1993. Sciences et intérêts : la figure de la dénonciation. À propos d'un livre d'Y. Lenoir sur l'effet de serre, Natures Sciences Sociétés, 1, 3, 238-245.

Godard, O., 1995. L'environnement, du champ de recherche au concept. Une hiérarchie enchevêtrée dans la formation du sens, Revue internationale de systémique, 9, 4, 405-428.

Godard, O., 1997. Le concept d'environnement, une hiérarchie enchevêtrée, in Larrère, C., Larrère, R. (Eds), La Crise environnementale, Paris, INRA Éditions, 97-112.

Godard, O., 2001. L'expertise économique du changement climatique planétaire. 1. Modèles d'organisation de l'expertise. 2. Sur la scène internationale, le GIEC. 3. Sur la scène française, autour du Commissariat général du Plan, Les Annales des Mines / Responsabilité E Environnement, 21, 2365.

Godard, O., 2007a. Est-il légitime de soumettre la connaissance scientifique au débat public? Les Annales des mines / Réalités industrielles, mai, 12-18.

Godard, O., 2007b. Le Rapport Stern sur l'économie du changement climatique était-il une manipulation grossière de la méthodologie économique? Revue d'économie politique, 117, 4, 475-506.

Godard, O., 2008a. Climate modelling for policy-making: How to represent freedom of choice and concern for future generations? Interdisciplinary Science Reviews, 33, 1, 51-69.

Godard, O., 2008b. Long terme et actualisation. La controverse suscitée par le rapport Stern sur le changement climatique, Revue de philosophie économique, 9, 2, 69-91. 
Godard, O., 2010. De l'imposture au sophisme, la science du climat vue par Claude Allègre, François Ewald et quelques autres, Esprit, mai, 26-43.

Gosseries, A., 2006. Égalitarisme cosmopolite et effet de serre, Paris, IDDRI.

Hallegatte, S., 2008. Adaptation to Climate Change: Do Not Count on Climate Scientists to Do Your Work, Washington, DC, RegMarkets Center.

Hallegatte, S., Ghil, M., 2008. Natural disasters impacting a macroeconomic model with endogenous dynamics, Ecological Economics, 68, 1-2, 582-592.

Hansen, J, Sato, M., Kharecha, P., Beerling, D., Berner, R., Masson-Delmotte, V., Pagani, M., Raymo, M., Royer, D.L., Zachos, J.C., 2008. Target atmospheric CO2: Where should humanity aim? Open Atmospheric Science Journal, 2, October 15, 217-231, doi : 10.2174/1874282300802010217.

Jamieson, D., 2005. Adaptation, mitigation and justice, in Sinnott-Armstrong, W., Howarth, R.B. (Eds), Perspectives on Climate Change: Sciences, Economics, Politics, Ethics, Oxford, Elsevier-JAI Press, 217-248.

Jepma, C.J., Munasinghe, M., 1998. Climate Change Policy: Facts, Issues and Analysis, Cambridge (UK), Cambridge University Press.

Lebeau, A., 2005. L'Engrenage de la technique : essai sur une menace planétaire, Paris, Gallimard.

Lebeau, A., 2008. L'Enfermement planétaire, Paris, Gallimard.

Lecocq, F., Shalizi, Z., 2007. Balancing Expenditures on Mitigation of and Adaptation to Climate Change: An Exploration of Issues Relevant to Developing Countries, Washington, DC, The World Bank, Development Research Group, Sustainable Rural and Urban Development Team, (Policy Research Working Paper 4299).

Lenoir, Y., 1992. La Vérité sur l'effet de serre : le dossier d'une manipulation planétaire, Paris, La Découverte.

Lewontin, R., 1978. L'adaptation, Pour la Science, 13.

Lewontin, R., 2000. The Triple Helix: Genes, Organisms and Environment, Cambridge (MA), Harvard University Press. Trad. fr. : La Triple hélice : les gènes, l'organisme, l'environnement, Paris, Le Seuil, 2003.

Lomborg, B., 2001. The Skeptical Environmentalist: Measuring the Real State of the World, Cambridge (UK), Cambridge University Press.

Magnan, A., Garnaud, B., Billé, R., Gemenne, F., Hallegatte, S. 2009. La Méditerranée au futur: des impacts du changement climatique aux enjeux de l'adaptation, Paris, IDDRI.

McMurry, A., 2003. Environmental Renaissance: On Emerson, Thoreau and Systems of Nature, Athens, University of Georgia Press.

Reçu le 24 juin 2009. Accepté le 1er juillet 2010.
Nordhaus, W., 1995. The ghosts of climates past and the specters of climate change future, Energy Policy, 23, 4-5, 269-282.

Nordhaus, W., 2007a. The Challenge of Global Warming: Economic Models and Environmental Policy, Manuscript, New Haven, Yale University.

Nordhaus, W., 2007b. A Review of the Stern Review on the economics of climate change, Journal of Economic Literature, $45,3,686-702$.

Parry, M., Lowe, J., Hanson, C., 2009. Overshoot, adapt and recover, Nature, 458, 1102-1103.

Pilkington, E., 2008. Climate target is not radical enough: Study, The Guardian, 7 April.

Ridley, M., 1996. Evolution, London, Blackwell Science.

Rittaud, B., 2010. Le Mythe climatique, Paris, Le Seuil.

Rogelj, J., Nabel, J., Chen, C., Hare, W., Markmann, K., Meinshausen, M., Schaeffer, W.M., Macey, K., Höhne, N., 2010. Copenhagen Accord pledges are paltry, Nature, 464, 1126-1128.

Rosenzweig, C., Parry, M.L., 1994. Potential impact of climate change on world food supply, Nature, 367, 133-138.

Seguin, B., 2007. Le réchauffement climatique et ses conséquences pour la viticulture. Communication au colloque Réchauffement climatique : quels impacts probables sur les vignobles? Dijon, Université de Bourgogne, 28-30 mars.

Schelling, T.C., 1997. The cost of combating global warming: Facing the tradeoffs, Foreign Affairs, 76, 6, 8-14.

Stern, N., 2007. The Economics of Climate Change: The Stern Review, Cambridge (UK), Cambridge University Press.

Sterner, T., Persson, U.M., 2008. An even Sterner Review: Introducing relative prices into the discounting debate, Review of Environmental Economics and Policy, 2, 1, 61-76.

Tol, R.S.J., 2005. Adaptation and mitigation: Trade-offs in substance and methods, Environmental Science and Policy, 8, 572578.

Tol, R.S.J., 2007. Europe's long-term climate target: A critical evaluation, Energy Policy, 35, 424-432.

Tol, R.S.J., Fankhauser, S., Smith, J.B., 1998. The scope for adaptation to climate change: What can we learn from the impact literature?, Global Environmental Change, 8, 2, 109-123.

Tol, R.S.J., Yohe, G.W., 2006. A Review of the Stern Review, World Economics, 7, 4, 233-250.

UNFCC, 2008. Investment and Financial Flows to Address Climate Change: An Update-Technical Paper. Doc FCCC/TP/2008/7, Geneva, 26 November.

Vivien, F.-D., 2005. Le Développement soutenable, Paris, La Découverte.

Weitzman, M.L., 2009. On modeling and interpreting the economics of catastrophic climate change, Review of Economics and Statistics, 91, 1, 1-19. 